\title{
Gender differences in doublecortin expression in the dentate gyrus of the Wistar rat during development
}

\author{
Özlem Tuğçe Çilingir Kaya ${ }^{1}$ (D), Sercan Doğukan Yıldız² (D), Nisva Hilal Levent ${ }^{1}$ (D), \\ Esra Bihter Gürler ${ }^{3}$ (D), Ümit Süleyman Şehirli² (D), Serap Sirvanc1 ${ }^{1}$ \\ ${ }^{1}$ Department of Histology and Embryology, School of Medicine, Marmara University, Istanbul, Turkey \\ ${ }^{2}$ Department of Anatomy, School of Medicine, Marmara University, Istanbul, Turkey \\ ${ }^{3}$ Department of Physiology, School of Medicine, Istanbul Atlas University, Istanbul, Turkey
}

\begin{abstract}
Objectives: Neurogenesis is the formation process of functional neurons from progenitor cells which continues during lifetime. Alterations in neurogenesis is associated with neurodegenerative disorders (ND). Different mechanisms underlie the ND in males and females which may be related to neurogenesis. In this study, we aimed to investigate the developmental process of neurogenesis in the hippocampus of male and female rats at different ages and shed light on the effect of gender difference on ND.

Methods: Brains were obtained from 7, 14, 21 days and 3-month-old male and female Wistar rats following intracardiac perfusion and processed for immunohistochemical and immunoflorescence staining. Doublecortin protein (DCX) was used as a marker of newly-born neuroblasts to determine neurogenesis.

Results: DCX immunoreactive (-ir) cells were dispersed throughout the granular and subgranular layers of DG in 7-days-old group in both genders. However, in the 14 and 21 days old groups, DCX-ir cells were observed only in the subgranular zone in the sections labelled with both immunohistochemistry (IHC) and immunoflourescent (IF) methods. In all age groups, female rats had a tendency to increase in DCX immunoreactivity when compared to that of male Wistar rats.

Conclusion: DCX-ir cells may be localized in different parts of DG during development. The number of newly born neurons showed a tendency to increase in female rats in all groups. Further studies are needed to understand the reason for differences in the normal developmental neurogenesis process between two genders.
\end{abstract}

Keywords: development; dentate gyrus; doublecortin; gender; neurogenesis

Anatomy 2020;14(2):111-116 @2020 Turkish Society of Anatomy and Clinical Anatomy (TSACA)

\section{Introduction}

Neurogenesis is the process of formation of functional neurons from progenitor cells. ${ }^{[1]}$ The phenomenon, which is known as only occuring in embryonic and perinatal periods, was shown in the hippocampal dentate granular cells of postnatal rats by Altman et al. ${ }^{[2]}$ Then, the newly born cells were shown to entegrate the local parts in the central nervous sytem. ${ }^{[3,4]}$ Under normal conditions, neurogenesis continues in the adult brain in the subgranular zone (SGZ) of hippocampal dentate gyrus (DG) and subventricular zone (SVZ) of lateral ventricles named as neurogenic regions. ${ }^{[5,6]}$ And, it is known that the level of neurogenesis is decreased by aging. ${ }^{[2]}$ Neurogenesis shows some similarities and differences in embryonic and adult periods. The most important difference is that the process occurs slower in adults when compared to the embryonic period. ${ }^{[7,8]}$

This study was partly presented as a poster at Neurocience 2018, Society for Neuroscience Meeting, 3-7 November 2018, San Diego, USA, and as an oral presentation at the International Mediterrenean Anatomy Congress, 6-9 September 2018, Istanbul, Turkey. 
Doublecortin protein (DCX) is a microtubule-associated protein which has an important role in the migration of neurons. It is needed for the stabilization and organization of microtubules. It is generally secreted by the migrating neurons of peripheral and central nervous system during embryonic and postnatal developmen. ${ }^{[9,10]}$ DCX is expressed by newly formed neuroblasts, thus, it is commonly used as a marker for determination of neurogenesis. In rodent nervous system, DCX is represented by the fast dividing progenitor cells during 30 days and ends up with neuronal maturation. ${ }^{[1]}$

In recent studies, gender differences attracted the attention not only as an effective factor on several brain regions and functions (emotion, memory, learning, navigation, levels of neurotransmitters etc.), but also on neurological disorders that have different mechanisms and incidences depending on the gender. ${ }^{[12-15]}$ Animal studies also have shown that immune system ${ }^{[16]}$ and prenatal and sex hormones ${ }^{[17-19]}$ have roles on brain development. For example, in male rats, acute stress suppresses adult neurogenesis, but not in female rats. ${ }^{[20,21]}$

Hippocampus is sexually dimorphic and directly related with learning and memory functions which are affected by the sex hormones. ${ }^{[2]}$ Neuroimaging studies have shown that the hippocampus is proportionally bigger in females in comparison to that inmales. ${ }^{[23]}$ Besides, in animal studies, a number of microscopical differences such as the structure of pyramidal cells in CA1 and the increased density of neurons in DG of males were observed. ${ }^{[24]}$ It is known that sex hormones such as estrogen have an effect on the excitability of hippocampal cells ${ }^{[25]}$ and the formation of dendritic structures. ${ }^{[26]}$

There is a gap in the literature examining the effects of gender differences on neurogenesis developmentally. Although the effects of gender in the CNS is known, most neuroscience studies are performed in male animals. Moreover, it has not been totally revealed how the sex hormones affect brain anatomy and cell morphology. To be able to make contribution to these issues, we aimed to examine the neurogenesis during developmental process by using 7, 14, 21-day-old and 3-month old male and female rats and determine the effects of gender on these processes by using two different methods at the light microscopic level.

\section{Materials and Methods}

Seven, 14, 21 days and 3 months old male and female Wistar rats ( $\mathrm{n}=5$, each; non-breeding) obtained from Experimental Animal Research and Implementation Center of Marmara University (DEHAMER) were used in the study. The animals were housed in a temperature-con- trolled room $\left(20 \pm 3^{\circ} \mathrm{C}\right)$ with a 12 -h light/dark cycle and fed on a standard diet. Full approval of the experimental procedures was obtained from the Animal Care and Use Committee of Marmara University (66.2017.mar, 11.09.2017).

Animals were deeply anesthetized with ketamine (100 $\mathrm{mg} / \mathrm{kg})$ and xylazine hydrochloride $(10 \mathrm{mg} / \mathrm{kg})$ and then euthanized by intracardiac perfusion with $4 \%$ paraformaldehyde in 0.1 M HEPES buffer, $\mathrm{pH}$ 7.4. The entire brains were removed and left overnight in the same fixative at $4^{\circ} \mathrm{C}$. All brain tissues underwent routine histological assesment. Briefly, brain tissues were dehydrated in ascending series of ethanol $(70,90,96,100 \%)$, cleared by xylene and embedded in paraffin. The brains were sectioned by a rotary microtome (Leica RM2125RT, Leica Biosystems, Nussloch, Germany) and labelled for DCX by both immunohistochemical and immunofluorescence methods. First, the sections were deparaffinized and then blocked with $0.06 \% \mathrm{H}_{2} \mathrm{O}_{2}$ solution. For the IHC labelling, blocked sections were incubated in $10 \%$ goat serum and $0.1 \%$ Triton X-100 solution in PBS buffer for $1 \mathrm{hr}$ and labelled with anti-DCX antibody (ab18723, Abcam, Cambridge, UK). Following washing with PBS, sections were incubated in a biotinylated secondary antibody (ab80437, Abcam, Cambridge, UK) for $30 \mathrm{~min}$ in room temperature. For light microscopic imaging, 3,3'diaminobenzidine (DAB) was used as a chromogen, whereas DyLight-550 conjugated secondary antibody (ThermoFisher Scientific, Waltham, MA, USA) was used for fluorescence microscopical imaging. Stained sections were examined by a brightfield and flourescence microscope (BX-51, Olympus Lifescience, Tokyo, Japan) attached to a CCD camera (DP72, Olympus Lifescience, Tokyo, Japan).

For quantitative analysis, hippocampal regions of brain tissues were evalauted in each section. DCX immunoreactive (DCX-ir) cell counting was performed on the micrographs which were taken randomly from 5 similar areas of each section at $\times 40$ magnification. ${ }^{[27]} \mathrm{We}$ also measured the granular cell layer (GCL) thickness of all experimental groups with ImageJ program (NIH). In each section, the thickness of the GCL was measured from 3 different regions and the mean values were used to perform statistical analyses. Statistical analyses were done by using GraphPad Prism 6 (v.6.07, San Diego, CA, USA). Data were analysed using one-way ANOVA and Tukey tests.

\section{Results}

Hippocampal regions of brain sections were observed in coherent morphology during developmental time courses.

In the 7, 14 and 21-day old groups, there was no difference in the total brain weights of female and male rats, 


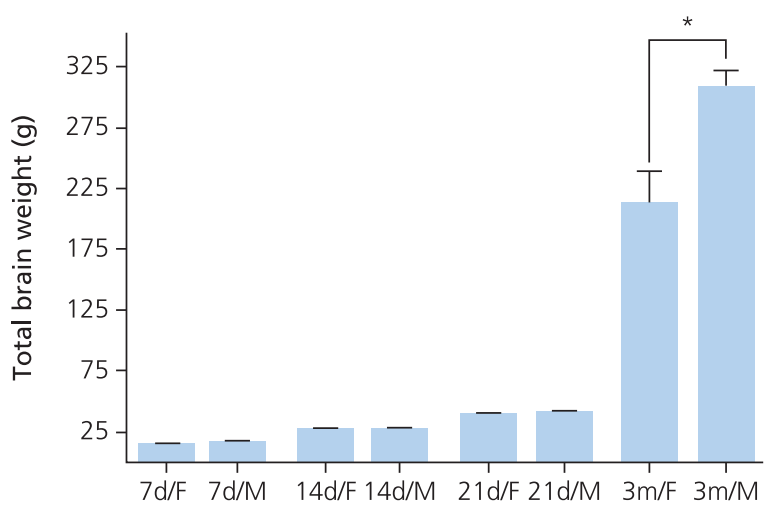

Figure 1. Comparative analysis of total brain weights of all experimental groups. d: day; m: month; F: female; M: male. ${ }^{*} p<0.0005$.

whereas, in the 3-month old group male brains were heav$\operatorname{ier}(\mathrm{p}<0.0005)$ when compared to female brains (Figure 1).

Because of morphological examination of the sections by light microscopy, DCX-ir cells were dispersed throughout the granular and subgranular layers of the hippocampal dentate gyri in 7 days old group in both female and male rats. However, in 14 and 21-day-old groups, DCX-ir cells were observed only in the subgranular zone in the sections labelled both with the two different types of staining methods. In accordance with the previous studies, DCX-ir cells were localized mostly in the subgranular layer in adult 3-month-old groups (Figures 2 and 3).

Additionally, morphological features were similar in the hippocampal cells in 7, 14, 21-day-old, and 3-monthold groups of female and male rats. With further morphological analyses, dentate granular cell layer were observed to be thicker in 3-month-old groups in comparison with the other age groups of both female and male rats, however, this difference was not statistically significant.

To compare the DCX-ir levels of female and male groups in different ages, a semiquantitative scoring method was applied to all sections. The result of this evaluation showed that the difference of the DCX-ir levels between the experimental groups was not statistically significant. However, it was perceived that DCX-ir cell numbers had a tendency to increase in female rats of all age groups. Furthermore, the lowest neurogenesis levels were observed in the 3-month-old groups of both female and male rats (Figure 4).

The data obtained from the GCL thickness measurements showed that in all experimental groups the thickness of the GCL increased when compared to that in females. However, this increase was statistically significant $(\mathrm{p}<0.05)$ in only the 3-month-old group (Figure 5).
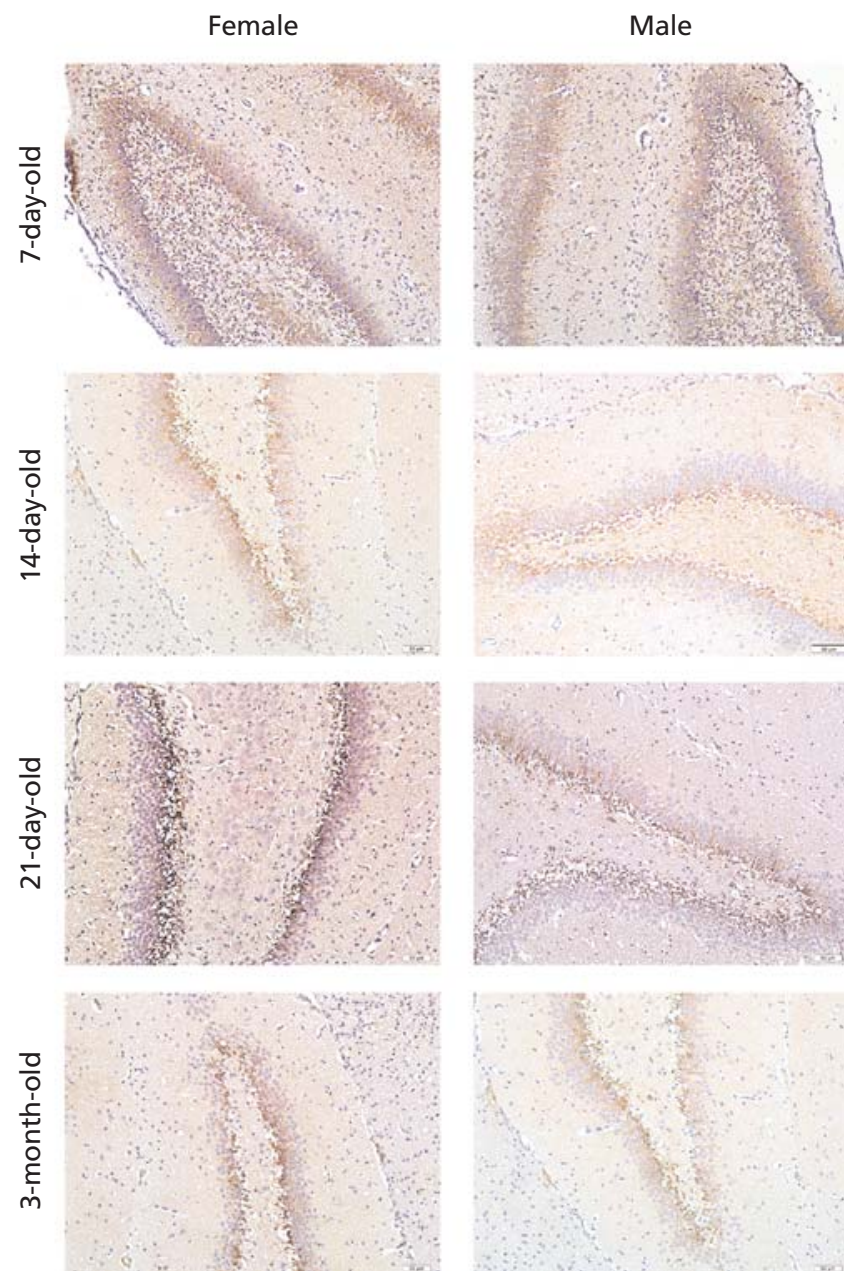

Figure 2. Micrographs of all experimental groups which were labelled for anti-DCX antibody immunohistochemically. Brown stained areas indicate DCX-ir cells. Bars: $50 \mu \mathrm{m}$.

\section{Discussion}

In the present study, we aimed to evaluate not only the developmental neurogenesis process, but also the effect of gender differences on this process at the light microscopic level.

Although the brain weights were not different in the 7, 14 and 21-day-old groups of female and male rats, in the 3-month-old groups male rats brains were significantly heavier than that of female rats. The relation between age, sex, maternal factors and brain weight was shown in previous studies. After reaching the sexual maturity, the weight of the brain strongly correlates with the body weight. ${ }^{[28]}$ Thus, it is an expected situation that adult male rats would have increased brain weights while there is no significant difference during developmental stages. 

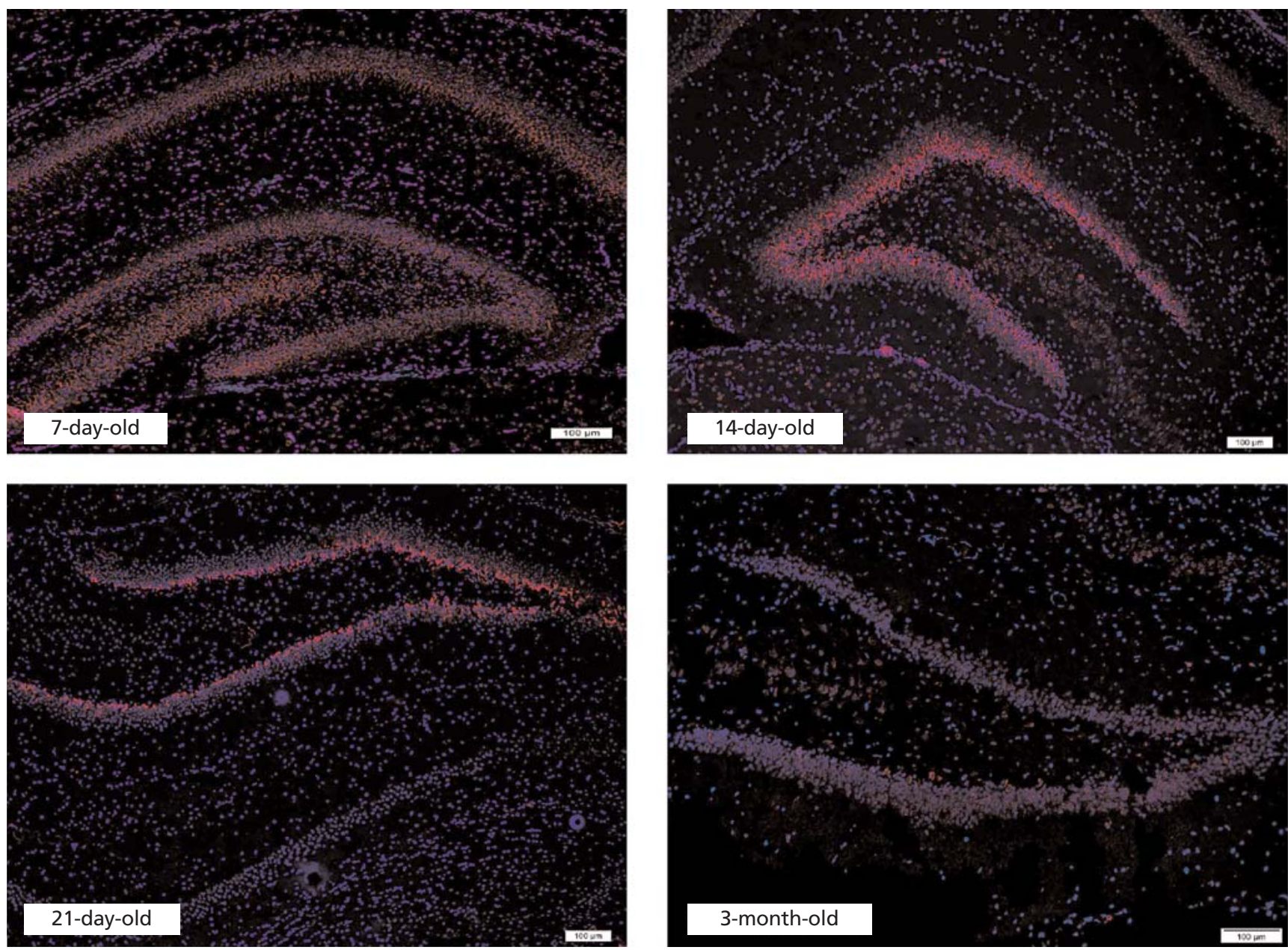

Figure 3. Immunoflorescence labelled micrographs for DCX immunoreactivity. Red staining indicates DCX-ir cells and blue staining indicates cell nuclei. Bars: $100 \mu \mathrm{m}$.

We observed that the granular cell layer was thicker in male dentate gyrus than that of the females in all groups however; the difference was statistically signifi-

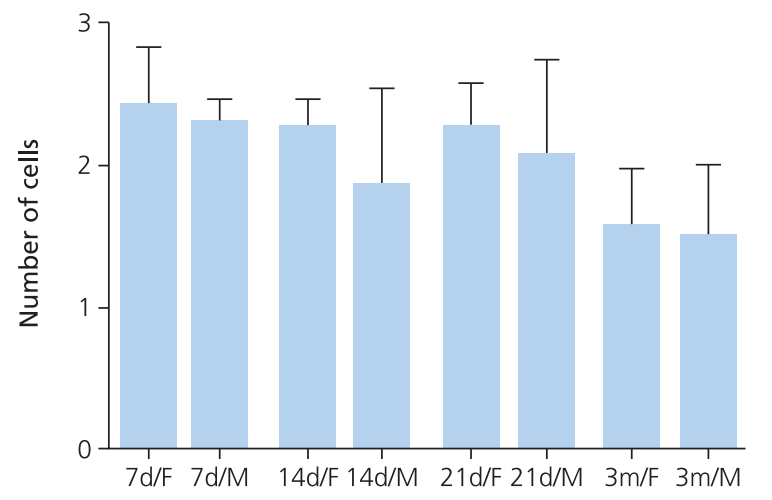

Figure 4. Semiquantitative analysis of DCX-ir cell number of all experimental groups. d: day; m: month; F: female; M: male. cant in only 3-months-old group. In a previous study, similar results were found that the volume of the cell layers within the dentate gyrus (hilus and granule cell

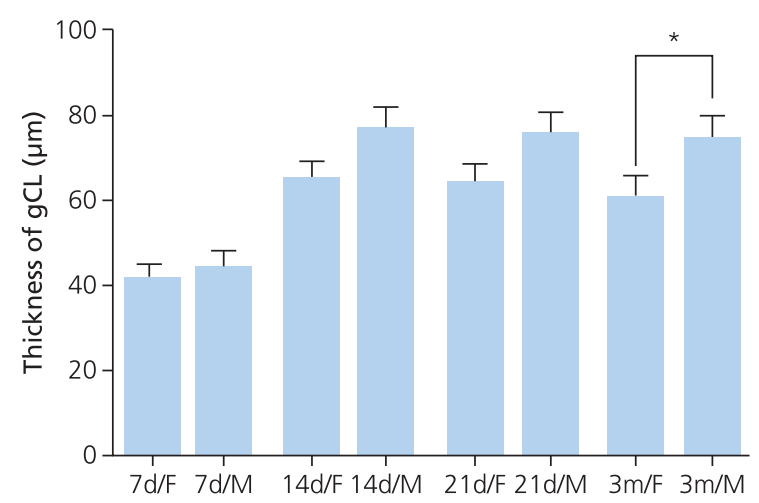

Figure 5. Statistical analyses of the granular cell layer $(G C L)$ thickness in all experimental groups. d: day; m: month; F: female; M: male. ${ }^{\star} p<0.05$. 
layer) was significantly larger in males than that of the females. ${ }^{[29]}$

According to the data from this study, DCX-ir cells are localized in different parts of the dentate gyrus during developmental process in both female and male rats. It was shown in previous studies that early immature granule cells expressed DCX as a marker of migration and dendritic growth, but the releasing turned off before neurons reach maturity. ${ }^{[30]}$ Since DCX is secreted by different migrating cell populations during development, ${ }^{[9]}$ it is normal to see DCX-ir at different regions of the DG. In a recent study, less differentiated DCX-ir cells were located closer to the subgranular zone, whereas more differentiated cells were located deeper within the granule cell layer and displayed morphological characteristics related to more mature granule cells. ${ }^{[31]}$ Further studies are needed in order to understand the mechanism by which the cells at the different levels of the granular layer in 7-day-old rats end up to be localized at the subgranular layer in later stages of development.

In a previous study which was done using wild-caught meadow voles, it was shown that the cell proliferation and cell death within the hippocampus were greater in nonbreeding than in breeding voles. ${ }^{[29]}$ In the present study, we used non-breeding rats to eleminate this difference in the neurogenesis levels.

It is a known reality that neurogenesis decreases with aging. ${ }^{[2,7,8]}$ Consistent with previous studies, in the present study, the lowest number of DCX-ir neurons were observed in the 3-month-old groups of both genders.

As a result of assesments of both IHC and IF staining methods, although a significant difference in the level of DCX immunoreactivity was not distinguished, it is a remarkable result that the DCX-ir cell numbers had a tendency to increase in female rats of all age groups. In the study of Spritzer et al., although male voles had larger hippocampal volumes than that of the females, the density of pHisH3-labeled cells (newly born cells) was found in greater amounts in the females compared to that in the males. ${ }^{[29]}$ In the same study, four different markers were used for showing the proliferative cells, including DCX. But the increase in the DCX-ir cell numbers of female voles was not statistically significant in comparison with that of the males, which is in consistent with our results in the present study. The results of another study suggest that male Wistar rats have more immature neurons (DCX-ir), compared to that of the females in adulthood. ${ }^{[21]}$ This study is also in line with our results, showing that there was no significant difference in the levels of DCX immunoreactivity between adut male and female rats.

\section{Conclusion}

The results suggested by the present study will contribute to the lack of data in this area and constitute the basis for both clinicians and researchers. We think that our study will form a ground for the further studies in the related area. However, the underlying mechanisms of developmental neurogenesis process in different genders is complex and require further clarifications.

\section{Conflict of Interest}

Authors declared no conflict of interest.

\section{Author Contributions}

OTCK: design of the work, performing research, data collection and analysis, writing the manuscript; SDY: design of the work, performing research; NHL: performing research; EBG: writing the manuscript; ÜSŞ: scientific advising; SS: scientific advising.

\section{Ethics Approval}

The study was approved by the Animal Care and Use Committee of Marmara University (66.2017.mar7, 11.09. 2017).

\section{Funding}

This study was supported by Research Fund of the Marmara University (grant number: SAG-K-081117-0608).

\section{References}

1. Ming GL, Song H. Adult neurogenesis in the mammalian brain: significant answers and significant questions. Neuron 2011;70:687-702.

2. Altman J, Das GD. Autoradiographic and histological evidence of postnatal hippocampal neurogenesis in rats. J Comp Neurol 1965;124: 319-35.

3. Paton JA, Nottebohm FN. Neurons generated in the adult brain are recruited into functional circuits. Science 1984;225:1046-8.

4. Reynolds BA, Weiss S. Generation of neurons and astrocytes from isolated cells of the adult mammalian central nervous system. Science 1992;255:1707-10.

5. Eriksson PS, Perfilieva E, Björk-Eriksson T, Alborn AM, Nordborg C, Peterson DA, Gage FH. Neurogenesis in the adult human hippocampus. Nat Med 1998;4:1313-7.

6. Gage FH. Mammalian neural stem cells. Science 2000;287:1433-8.

7. Zhao C, Teng EM, Summers RG, Jr., Ming GL, Gage FH. Distinct morphological stages of dentate granule neuron maturation in the adult mouse hippocampus. J Neurosci 2006;26:3-11.

8. Kempermann G, Jessberger S, Steiner B, Kronenberg G. Milestones of neuronal development in the adult hippocampus. Trends Neurosci 2004;27:447-52.

9. Gleeson JG, Lin PT, Flanagan LA, Walsh CA. Doublecortin is a microtubule-associated protein and is expressed widely by migrating neurons. Neuron 1999;23:257-71. 
10. Plumpe T, Ehninger D, Steiner B, Klempin F, Jessberger S, Brandt M, Römer B, Rodrigues GR, Kronenberg G, Kempermann G. Variability of doublecortin-associated dendrite maturation in adult hippocampal neurogenesis is independent of the regulation of precursor cell proliferation. BMC Neurosci 2006;7:77.

11. Brown JP, Couillard-Despres S, Cooper-Kuhn CM, Winkler J, Aigner L, Kuhn HG. Transient expression of doublecortin during adult neurogenesis. J Comp Neurol 2003;467:1-10.

12. Levine S. Sex differences in the brain. Scientific American 1966;214: 84-90.

13. Sacher J, Neumann J, Okon-Singer H, Gotowiec S, Villringer A. Sexual dimorphism in the human brain: evidence from neuroimaging. Magn Reson Imaging 2013;31:366-75.

14. Bao AM, Swaab DF. Sex differences in the brain, behavior, and neuropsychiatric disorders. Neuroscientist 2010;16:550-65.

15. Baron-Cohen S, Lombardo MV, Auyeung B, Ashwin E, Chakrabarti $\mathrm{B}$, Knickmeyer R. Why are autism spectrum conditions more prevalent in males? PLoS Biol 2011;9:e1001081.

16. Lenz KM, Nugent BM, Haliyur R, McCarthy MM. Microglia are essential to masculinization of brain and behavior. J Neurosci 2013; 33:2761-72.

17. Arnold AP, Chen X. What does the "four core genotypes" mouse model tell us about sex differences in the brain and other tissues? Front Neuroendocrinol 2009;30:1-9.

18. Phoenix CH. Organizing action of prenatally administered testosterone propionate on the tissues mediating mating behavior in the female guinea pig. Horm Behav 2009;55:566.

19. Arnold AP, Breedlove SM. Organizational and activational effects of sex steroids on brain and behavior: a reanalysis. Horm Behav 1985; 19:469-98.

20. Falconer EM, Galea LA. Sex differences in cell proliferation, cell death and defensive behavior following acute predator odor stress in adult rats. Brain Res 2003;975:22-36.

21. Hillerer KM, Neumann ID, Couillard-Despres S, Aigner L, Slattery DA. Sex-dependent regulation of hippocampal neurogenesis under basal and chronic stress conditions in rats. Hippocampus 2013;23: 476-87.

22. Cahill L. Why sex matters for neuroscience. Nature Rev Neurosci 2006;7:477-84.

23. Goldstein JM, Seidman LJ, Horton NJ, Makris N, Kennedy DN, Caviness VS Jr, Faraone SV, Tsuang MT. Normal sexual dimorphism of the adult human brain assessed by in vivo magnetic resonance imaging. Cereb Cortex 2001;11:490-7.

24. Madeira MD, Lieberman AR. Sexual dimorphism in the mammalian limbic system. Prog Neurobiol 1995;45:275-333.

25. Teyler TJ, Vardaris RM, Lewis D, Rawitch AB. Gonadal steroids: effects on excitability of hippocampal pyramidal cells. Science 1980; 209:1017-8.

26. Cooke BM, Woolley CS. Gonadal hormone modulation of dendrites in the mammalian CNS. J Neurobiol 2005;64:34-46.

27. Pazar A, Kolgazi M, Memisoglu A, Bahadir E, Sirvanci S, Yaman A, Yeğen BÇ, Ozek E. The neuroprotective and anti-apoptotic effects of melatonin on hemolytic hyperbilirubinemia-induced oxidative brain damage. J Pineal Res 2016;60:74-83.

28. Goffinet AM, Rakic P. Mouse brain development. Results and problems in cell differentiation. Heidelberg: Springer; 2000.

29. Spritzer MD, Panning AW, Engelman SM, Prince WT, Casler AE, Georgakas JE, Jaeger ECB, Nelson LR, Roy EA, Wagner BA. Seasonal and sex differences in cell proliferation, neurogenesis, and cell death within the dentate gyrus of adult wild-caught meadow voles. Neuroscience 2017;360:155-65.

30. Spampanato J, Sullivan RK, Turpin FR, Bartlett PF, Sah P. Properties of doublecortin expressing neurons in the adult mouse dentate gyrus. PloS One 2012;7:e41029.

31. Moreno-Jiménez EP, Flor-García M, Terreros-Roncal J, Rábano A, Cafini F, Pallas-Bazarra N, Avila J, Llorens-Martin M. Adult hippocampal neurogenesis is abundant in neurologically healthy subjects and drops sharply in patients with Alzheimer's disease. Nat Med 2019;25:554-60.

Correspondence to: Özlem Tuğçe Çilingir Kaya, PhD Department of Histology and Embryology, School of Medicine, Marmara University, Maltepe, Istanbul, Turkey Phone: +90 2167775562 e-mail: tugce.cilingir@marmara.edu.tr Conflict of interest statement: No conflicts declared. ND4.0) Licence (http://creativecommons.org/licenses/by-nc-nd/4.0/) which permits unrestricted noncommercial use, distribution, and reproduction in any medium, provided the original work is properly cited. How to cite this article: Çilingir Kaya ÖT, Yıldız SD, Levent NH, Gürler EB, Şehirli ÜS, Sirvancı S. Gender differences in doublecortin expression in the dentate gyrus of the Wistar rat during development. Anatomy 2020;14(2):111-116. 